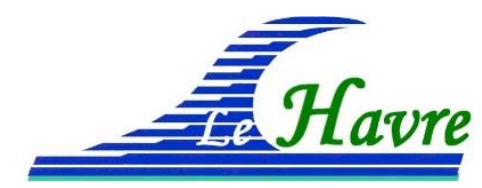

XVIèmes Journées Nationales Génie Côtier - Génie Civil
Le Havre, 2020

DOI:10.5150/jngcgc.2020.029 ～(C) Editions Paralia CFL

disponible en ligne - http://www.paralia.fr - available online

\title{
Processus d'affouillement au pied d'un mur vertical de protection côtière
}

\section{Laurence MAROIS ${ }^{1}$, Jacob STOLLE ${ }^{1}$, Damien PHAM VAN BANG ${ }^{1}$}

1. Institut National de la Recherche Scientifique (INRS), Université du Québec, Eau, Terre et Environnement (ETE), 2605 Bld du Parc Technologique, G1P 4S5, Québec (QC). damien.pham_van_bang@ete.inrs.ca

\section{Résumé :}

Au Québec, plusieurs murs verticaux ont été construits pour protéger le trait de côte. Un affouillement peut se développer au pied de ces ouvrages et menacer leur stabilité. Une campagne expérimentale en canal à houle a été récemment menée pour étudier l'affouillement au pied d'un mur vertical simulant une protection côtière. L'étude sous conditions régulières de vagues est réalisée sous différentes conditions hydrosédimentaires. Les mesures portent sur le suivi de la surface libre, des vitesses à proximité du mur et de l'évolution morphodynamique du lit sédimentaire à proximité de l'ouvrage. Une méthode à quatre sondes fixes est appliquée pour séparer les composantes incidentes et réfléchies de la houle, la technique de PLIF pour mesurer les vitesses et une technique acoustique pour accéder à la bathymétrie. Les conditions hydrodynamiques (profondeur d'eau, période, hauteur de vague) et sédimentaires (diamètre, pente) sont étudiées, de même que les caractéristiques de l'ouvrage (position, géométrie). L'évolution temporelle de l'affouillement au pied du mur suit une tendance exponentielle. Différents modes d'affouillement ont été observés.

Mots-clés : Expérimentation, Génie côtier, Hydraulique maritime, Sédiments, Ouvrages de protection, Affouillement au pied d'ouvrage.

\section{Introduction}

Les territoires en bordure de mer présentent de nombreux enjeux socio-économiques. Par exemple, un tiers de la population et près de $60 \%$ des routes nationales sont situés à moins de $500 \mathrm{~m}$ des côtes dans les régions du Bas-Saint-Laurent-Gaspésie-Iles de la Madeleine au Québec. Et différents ouvrages de protection ont été construits pour lutter contre l'érosion. La présente étude porte sur les ouvrages côtiers de type mur vertical. Ces derniers sont très réflectifs et modifient fortement l'hydrodynamique, donc le transport sédimentaire. Ils induisent de fortes redirections verticales de l'écoulement : les jets de paroi ascendants créent des splash; les jets descendants peuvent affouiller le lit sédimentaire sur lequel est fondé l'ouvrage, donc conduire à l'effondrement de la protection par diminution de la capacité portante du sol (WHITEHOUSE, 1998). Bien que ces ouvrages soient anciens et aient beaucoup été étudiés par le passé, les formules actuellement disponibles pour prédire l'affouillement restent imprécises. L'objectif de 


\section{Thème 2 - Dynamique sédimentaire}

cette étude est de renouveler la connaissance des processus d'affouillement au pied d'un mur vertical de protection côtière, à partir d'une approche expérimentale en canal à houle équipé d'une instrumentation à haute résolution. Les tests sont effectués pour différentes hauteurs d'eau, amplitude-période de vagues, pentes de plage et granulométrie de sédiments. Les mesures portent sur les caractéristiques des vagues, les champs de vitesses à proximité du mur au cours de l'évolution de l'affouillement. L'article propose une revue succincte des principales formules d'affouillement, puis une description des essais. Les résultats sont ensuite présentés avec des analyses. Une nomenclature de symboles utilisés est fournie dans le tableau 1 suivant.

Tableau 1. Nomenclature utilisée.

\begin{tabular}{lll}
\hline Symbole & Définition & Dimension \\
\hline$H$ & Hauteur de vague & $\mathrm{m}$ \\
$h$ & Hauteur d'eau & $\mathrm{m}$ \\
$h_{w}$ & Hauteur d'eau au mur & $\mathrm{m}$ \\
$L$ & Longueur d'onde & $\mathrm{m}$ \\
$S_{\max }$ & Affouillement maximal & $\mathrm{m}$ \\
$T$ & Période & $\mathrm{S}$ \\
$w_{s}$ & Vitesse de chute & $\mathrm{m} / \mathrm{s}$ \\
$X$ & Distance du mur au trait de côte naturel & $\mathrm{m}$ \\
$X_{b}$ & Distance du point de déferlement au trait de côte naturel & $\mathrm{m}$ \\
$\beta$ & Pente de la plage & \\
$d_{50}$ & Diamètre médian & $\mu \mathrm{m}$ \\
$\xi_{0}$ & Nombre d'Iribaren & \\
$\Omega$ & Nombre de Dean & \\
\hline
\end{tabular}

\section{Méthode expérimentale et matériaux}

Les expérimentations ont été réalisées dans le petit canal à houle (section $0,3 \times 0,3 \mathrm{~m}^{2}$, longueur $7 \mathrm{~m}$ ) en plexiglas du Laboratoire Hydraulique Environnemental de l'INRS (http://lhe.ete.inrs.ca/). Les vagues sont générées par un batteur pouvant opérer soit en mode piston soit en mode volet. Dans cette étude, le mode piston est sélectionné et un logiciel (environnement Labview(C) a été développé pour imposer son mouvement : dans le cas d'une houle régulière, les amplitudes et périodes de vagues sont parfaitement contrôlées. La zone d'étude s'étend sur les deux derniers mètres du canal (figure 1). Une plage est réalisée sur $1 \mathrm{~m}$ entre une pente rigide et un mur vertical lisse (plexiglas). Les sédiments sont déposés et la pente de la plage est réalisée à l'aide d'un dispositif de régalage. Trois tailles de sédiments et trois pentes sont considérées (tableau 2) dans les tests avec une hauteur d'eau, $h$, entre 10 et $16 \mathrm{~cm}$, une hauteur de vague, $H$, entre 1,3 et $2,5 \mathrm{~cm}$ et une période, $T$, entre 0,8 et $3 \mathrm{~s}$.

Trois campagnes d'essais ont été effectuées : la première avec du sable fin $\left(\mathrm{d}_{50}=216 \mu \mathrm{m}\right.$, $\left.\mathrm{W}_{\mathrm{s}}=1.86 \mathrm{~cm} / \mathrm{s}\right)$; la deuxième avec du sable moyen $\left(\mathrm{d}_{50}=525 \mu \mathrm{m}, \mathrm{w}_{\mathrm{s}}=2.9 \mathrm{~cm} / \mathrm{s}\right)$; la dernière avec des billes de verre $\left(\mathrm{d}_{50}=700 \mu \mathrm{m}, \mathrm{w}_{\mathrm{s}}=9 \mathrm{~cm} / \mathrm{s}\right)$. La granulométrie de ces matériaux est uniforme. 


\section{XVİ̀mes Journées Nationales Génie Côtier - Génie Civil \\ Le Havre 2020}

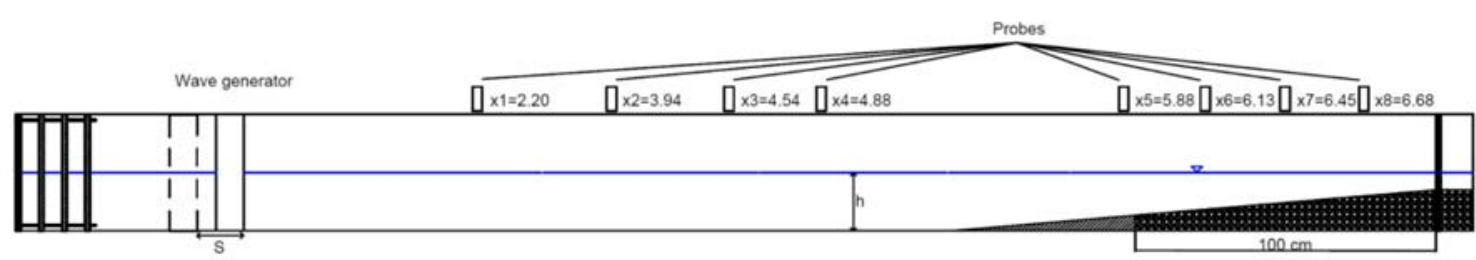

Figure 1. Schéma du montage : batteur-piston; pente rigide $(50 \mathrm{~cm}) ;$ plage $(100 \mathrm{~cm})$; mur vertical ; 8 sondes de niveaux d'eau (position à partir du batteur).

Tableau 2. Paramètres des 20 tests.

\begin{tabular}{llllll}
\hline Essai & $\boldsymbol{d}_{\mathbf{5 0}}(\boldsymbol{\mu m})$ & $\boldsymbol{h}(\mathbf{c m})$ & $\boldsymbol{H}(\mathbf{c m})$ & $\boldsymbol{T}(\mathbf{s})$ & $\boldsymbol{\beta}$ \\
\hline 1 & 216 & 13 & 1,7 & 1,5 & $1: 25$ \\
3 & 216 & 13 & 1,7 & 2 & $1: 25$ \\
4 & 216 & 15 & 2,5 & 0,8 & $1: 25$ \\
8 & 216 & 10 & 2,1 & 0,8 & $1: 100$ \\
10 & 216 & 10 & 1,5 & 2,5 & $1: 100$ \\
13 & 216 & 16 & 1,3 & 2 & $1: 10$ \\
14 & 216 & 16 & 1,7 & 1 & $1: 10$ \\
F1 & 525 & 10 & 2,1 & 0,8 & $1: 100$ \\
F4 & 525 & 10 & 1,7 & 2 & $1: 100$ \\
F6 & 525 & 13 & 2,2 & 2,5 & $1: 25$ \\
F7 & 525 & 13 & 1,7 & 2 & $1: 25$ \\
F9 & 525 & 15 & 1,6 & 3 & $1: 25$ \\
F10 & 525 & 15 & 2,5 & 1 & $1: 25$ \\
F12 & 525 & 16 & 1,3 & 2 & $1: 10$ \\
F13 & 525 & 16 & 1,7 & 1 & $1: 10$ \\
GB1 & 700 & 16 & 1,7 & 1 & $1: 10$ \\
GB2 & 700 & 16 & 1,3 & 2 & $1: 10$ \\
GB3 & 700 & 15 & 2,5 & 1 & $1: 25$ \\
GB4 & 700 & 15 & 1,6 & 3 & $1: 25$ \\
GB5 & 700 & 13 & 1,7 & 2 & $1: 25$ \\
\hline
\end{tabular}

Les caractéristiques des vagues sont mesurées en huit points par des sondes acoustiques (General Acoustic(C) USS3). Ces dernières sont adaptées aux mesures à petite échelle (plage de mesure entre 3 et $35 \mathrm{~cm}$ ) et à haute fréquence d'acquisition (entre 50 et $100 \mathrm{~Hz}$ ). Quatre sondes ont été placées au-dessus de la plage et quatre autres ont été réparties entre le batteur et la zone d'étude (figure 1).

Les propriétés 3D de l'écoulement sur la zone d'intérêt sont obtenues par vélocimétrie par image de particules en stéréo (LaVisionC), stéréo-PIV, 2D3C, 7Hz). La technique donne accès au champ 2D des 3 composantes de vitesse sur la zone d'étude. La technique PLIF (Planar Laser Induced Fluorescence) est mise au point pour réduire les problèmes de mesure liés à la réflexion du laser sur la surface libre, le mur en plexiglas ou le lit sédimentaire. Des traceurs fluorescents sont utilisés : ils sont fabriqués au laboratoire à partir de résine et de rhodamine WT suivant la méthode de PEDOCCHI et al. (2008).

L'évolution de l'affouillement est suivie par des relevés bathymétriques. Dans cette étude, un échographe Doppler médical (General Electric (C), LogiQ) est utilisé avec une 


\section{Thème 2 - Dynamique sédimentaire}

sonde linéaire à $12 \mathrm{MHz}$ (modèle 12L-RS) : il enregistre par imagerie acoustique les profils de plage toutes les 30 minutes. Des caméras vidéo permettent des mesures à plus haute fréquence (toutes les 5 minutes) en début d'essai.

\section{Résultats et discussions}

\subsection{Evolution temporelle de 1'affouillement}

La figure 2a présente l'évolution de l'affouillement avec le temps (essai 3) dans la zone d'étude, entre le début de la zone érodable $(x=100 \mathrm{~cm})$ et le mur $(x=0 \mathrm{~cm})$. Le lit initialement plat se déstabilise en formant des rides, l'érosion se localise sur le mur, mais aussi aux abords de la transition pente rigide-plage érodable $(\mathrm{x}=100 \mathrm{~cm})$. Le système forme une dune centrale avec une progression offshore. La profondeur maximale d'affouillement normalisée par la hauteur des vagues (figure 2b) évolue rapidement en début d'essai puis tend à saturer avec le nombre de vagues ou le temps.
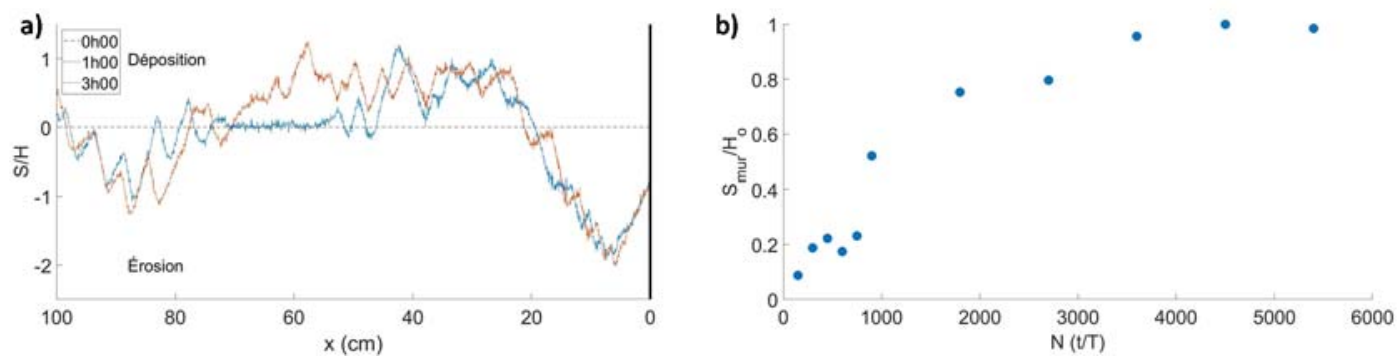

Figure 2. Évolution temporelle de l'affouillement (Essai 3) : a) profil de plage; b) profondeur d'affouillement.

La dynamique sur la profondeur d'affouillement (Fig. 2b) respecte la loi de décroissance exponentielle proposée par HERBICH et VAN WEELE (1965) à deux paramètres :

$$
S_{t}=S_{\text {max }}\left(1-\exp \left(-\frac{t}{\tau}\right)\right)
$$

où $S_{\max }$ est l'affouillement à l'équilibre et $\tau$ une constante de temps.

Si différentes formulations sont proposées pour relier $S_{\max }$ aux conditions de vagues, elles peuvent différer selon les paramètres d'entrée ou les configurations expérimentales. Par exemple, SONG \& SCHILLER (1973) expriment $S_{\max }$ en fonction de la cambrure des vagues $H / L$ et la position du mur $X_{s}$.

$$
\frac{S_{\max }}{H_{o}}=1,94+0,57 \ln \left(X_{S}\right)+0,72 \ln \left(\frac{H_{S}}{L_{S}}\right)
$$

où $X_{s}=X / X_{b}$ et l'indice $s$ se réfère à une hauteur significative.

JONES (1975) propose une relation empirique qui ne dépend que de la position du mur. Sa formule prédit un affouillement nul pour $X_{s}=1$, i.e. lorsque les vagues déferlent sur le mur, ce qui est démenti par les résultats de tests : 


\section{XVI'̀mes Journées Nationales Génie Côtier - Génie Civil \\ Le Havre 2020}

$$
\frac{S_{\max }}{H_{b}}=1,60\left(1-X_{S}\right)^{2 / 5}
$$

FOWLER (1992) relie l'affouillement à la profondeur d'eau au mur, $h_{w}$, adimensionalisée par la longueur d'onde loin du mur, $L_{0}$ :

$$
\frac{S_{\max }}{H_{o}}=\sqrt{22,72 \frac{h_{w}}{L_{o}}+0,25}
$$

Enfin, MCDOUGAL et al. (1996) présente à partir de simulations numériques (code $\mathrm{SBEACH}$ ) une formule faisant intervenir la pente initiale du lit, $\beta$, la cambrure, la hauteur d'eau au mur et la taille des sédiments, $d_{50}$ :

$$
\frac{S_{\max }}{H_{o}}=0,41 \beta^{0,85}\left(\frac{L_{o}}{H_{o}}\right)^{1 / 5}\left(\frac{h_{w}}{H_{o}}\right)^{1 / 4}\left(\frac{H_{o}}{D_{50}}\right)^{1 / 3}
$$

En revanche, au meilleur de nos connaissances, il n'existe pas de formulations pour la constante de temps, $\tau$, dans la formulation (1). Pour l'ensemble de nos tests, ce paramètre semble constant et égale à 30 minutes environ. Compte tenu de cette observation, la durée des tests est fixée à $180 \mathrm{~min}$ soit $3 \mathrm{~h}$. Si l'affouillement n'est pas observé après 30 minutes, le test est interrompu.

Les résultats sur $S_{\max }$ sont présentés en fonction de la cambrure des vagues (Fig. 3a) et de la hauteur d'eau au mur (Fig. 3b). La diminution de l'affouillement avec la cambrure est observée : cette tendance est en accord avec les relations (2) et (5). La relation (4) prédit une augmentation systématique de l'affouillement avec la hauteur d'eau au mur, $h_{w} / L_{0}$, ce qui n'est pas toujours vérifiée par nos résultats. Une diminution ; voire une accrétion, a été observée par PEARCE et al. (2006) aux fortes valeurs de $h_{w}$.
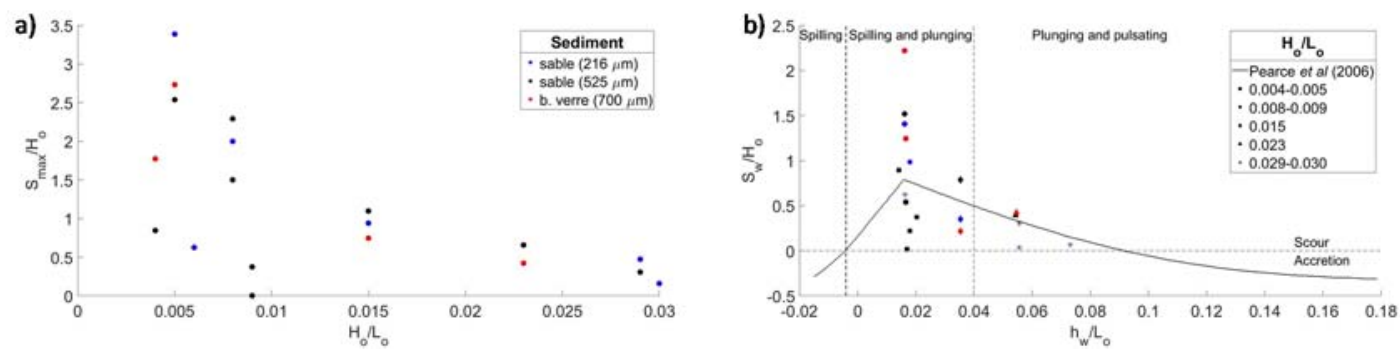

Figure 3. Evolution de l'affouillement en fonction de la cambrure (a) et de la hauteur d'eau au mur (b).

Le tableau 2 présente les cas pour lesquels un affouillement a été observé. Des cas avec accrétion ont été observés, ce qui est en accord avec les observations de PEARCE et al. (2006), mais ne sont pas retenus pour les analyses (tableau 2) de cette étude. Pour les cas avec affouillement, les effets de la pente initiale de la plage et de la taille des sédiments sur la profondeur maximale sont présentés sur les figure $4 \mathrm{a}$ et $4 \mathrm{~b}$ respectivement. L'augmentation (ou diminution) de $S_{\max }$ avec la pente $\beta$ (ou le diamètre des sédiments $d_{50}$ ) est observée, ce qui est conforme avec (5). 


\section{Thème 2 - Dynamique sédimentaire}
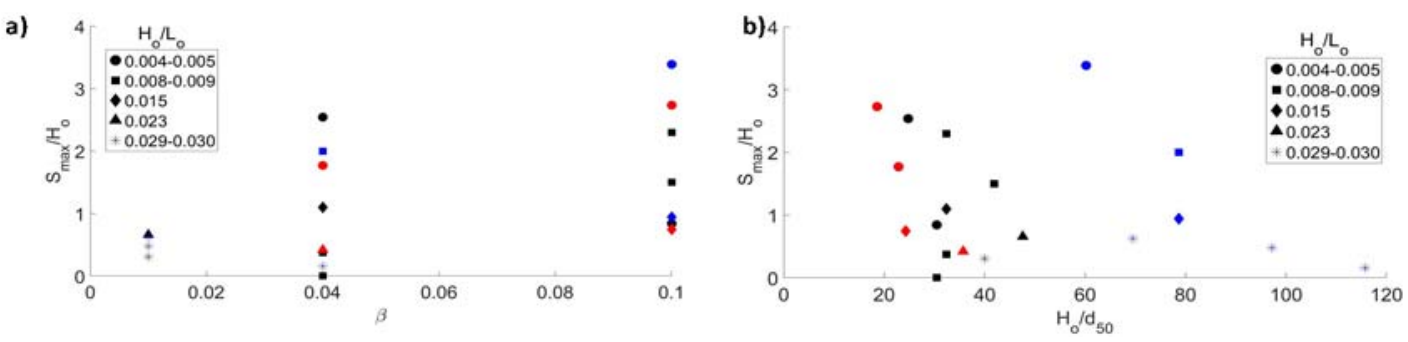

Figure 4. Evolution de la profondeur d'affouillement en fonction de la pente initiale de la plage (a) et du diamètre des sédiments (b).

\subsection{Champs de vitesse au voisinage de l'ouvrage}

Les mesures de vitesse par la technique de stéréo-PIV couplée à la méthode PLIF permettent d'accéder aux informations sur la surface libre, le mur et le lit sédimentaire (figure 5).
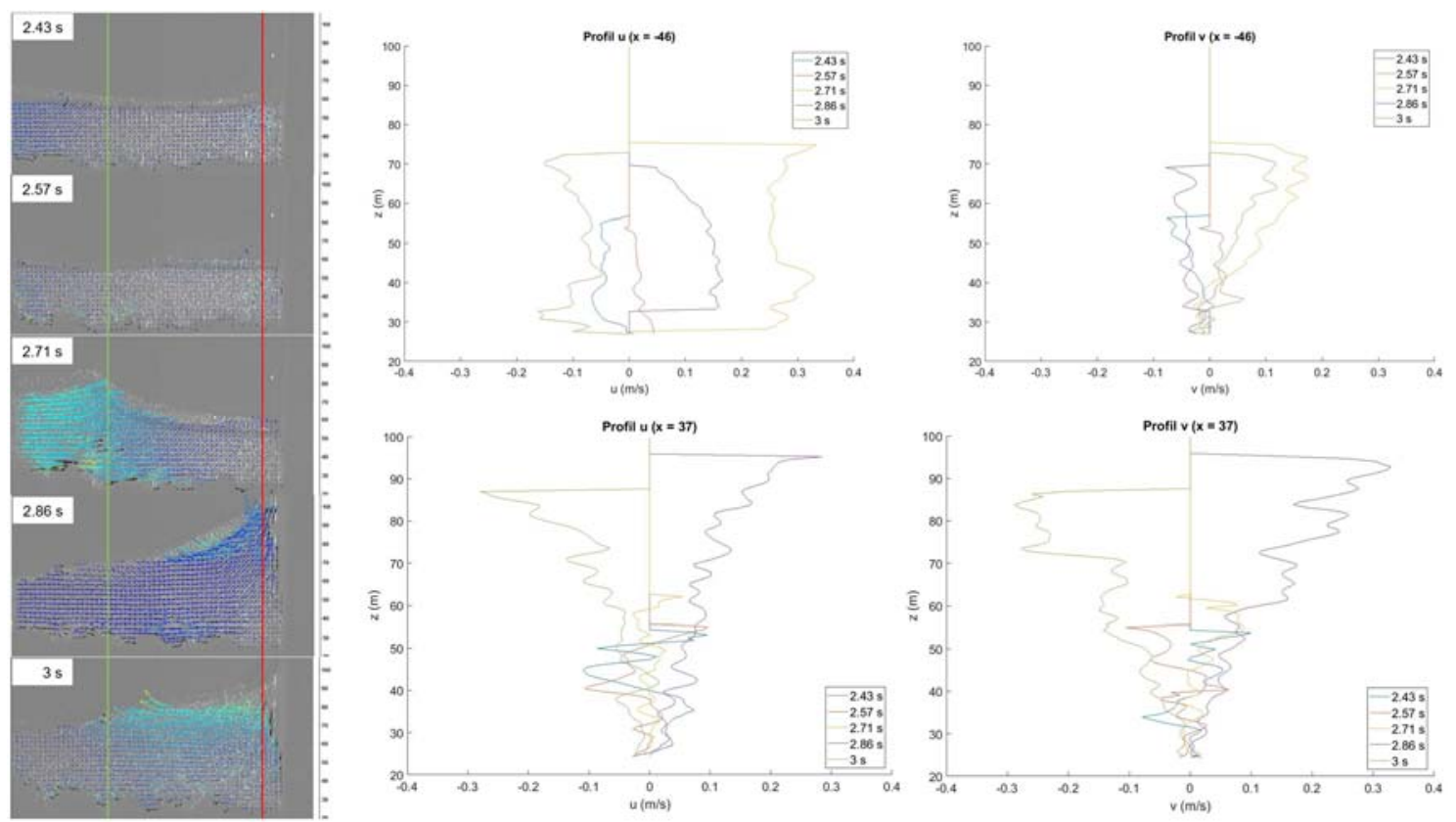

Figure 5. Imagerie et vélocimétrie pour un impact de vague (essai 3) : profils verticaux de vitesses horizontales et verticales à deux positions (lignes verte et rouge) du mur.

Pour un impact de vague, les observations mettent en évidence des alternances de vitesses horizontales dirigées ou opposées au mur, ainsi que des vitesses verticales (ou jets de paroi) ascensionnels ou plongeants. Contrairement aux configurations classiques des érosions avec un écoulement tangentiel, la présence de l'ouvrage génère une composante verticale à la vitesse qui impacte ou aspire le lit sédimentaire. 


\section{XVİ̀mes Journées Nationales Génie Côtier - Génie Civil \\ Le Havre 2020}

La présence du mur, de la plage et du batteur à houle ajoute une composante de réflexion à la houle et génère des harmoniques d'ordre supérieur. Afin de bien caractériser les conditions hydrodynamiques du canal, il est important de séparer les composantes incidentes et réfléchies des modes fondamental et harmonique. La méthode à quatre sondes fixes de LIN \& HUANG (2004) est considérée pour décomposer le signal selon : $\eta\left(x_{m}, t\right)=a_{I}^{(1)} \cos \left(k x_{m}-\omega t+\phi_{I}^{(1)}\right)+a_{R}^{(1)} \cos \left(k x_{m}+\omega t+\phi_{R}^{(1)}\right)+$ $\sum_{n \geq 2} a_{I, B}^{(n)} \cos \left[n\left(k x_{m}-\omega t\right)+\phi_{I, B}^{(n)}\right]+\sum_{n \geq 2} a_{R, B}^{(n)} \cos \left[n\left(k x_{m}+\omega t\right)+\phi_{R, B}^{(n)}\right]+$ $\sum_{n \geq 2} a_{I, F}^{(n)} \cos \left[k^{(n)} x_{m}-n \omega t+\phi_{I, F}^{(n)}\right]+\sum_{n \geq 2} a_{R, F}^{(n)} \cos \left[k^{(n)} x_{m}+n \omega t+\phi_{R, F}^{(n)}\right]+e_{m}$ où $a$ est l'amplitude, $\omega$ la fréquence angulaire, $k$ le nombres d'onde, $\phi$ le déphasage. Les indices $I$ et $R$ sont utilisés pour identifier les ondes incidentes et réfléchies; les indices $B$ et $F$ désignent les ondes liées (bound) et libre (free).

Les coefficients de réflexion linéaire $\left(\mathrm{Kr}^{(1)}\right)$ et non linéaire $\left(\mathrm{Kr}^{(2)}\right)$ peuvent aussi être calculés d'après les formules suivantes :

$$
K_{r}^{(1)}=\sqrt{\frac{\left(a_{R}^{(1)}\right)^{2}}{\left(a_{I}^{(1)}\right)^{2}}}, \quad K_{r}^{(2)}=\sqrt{\frac{\left(a_{R}^{(1)}\right)^{2}+\left(a_{R, B}{ }^{(2)}\right)^{2}+\left(a_{R, F}(2)\right)^{2}}{\left(a_{I}^{(1)}\right)^{2}+\left(a_{I, B}{ }^{(2)}\right)^{2}+\left(a_{I, F}{ }^{(2)}\right)^{2}}}
$$

Au cours d'un essai, les signaux des 4 sondes de vagues placées au-dessus de la plage (proche du mur, Fig. 1) sont décomposés en séquences de 5 minutes. Sur chacune d'elle, la méthode est appliquée pour déterminer les coefficients d'amplitude et de réflexion. L'évolution temporelle de ces paramètres est présentée sur la figure 6 correspondant à l'essai 1.
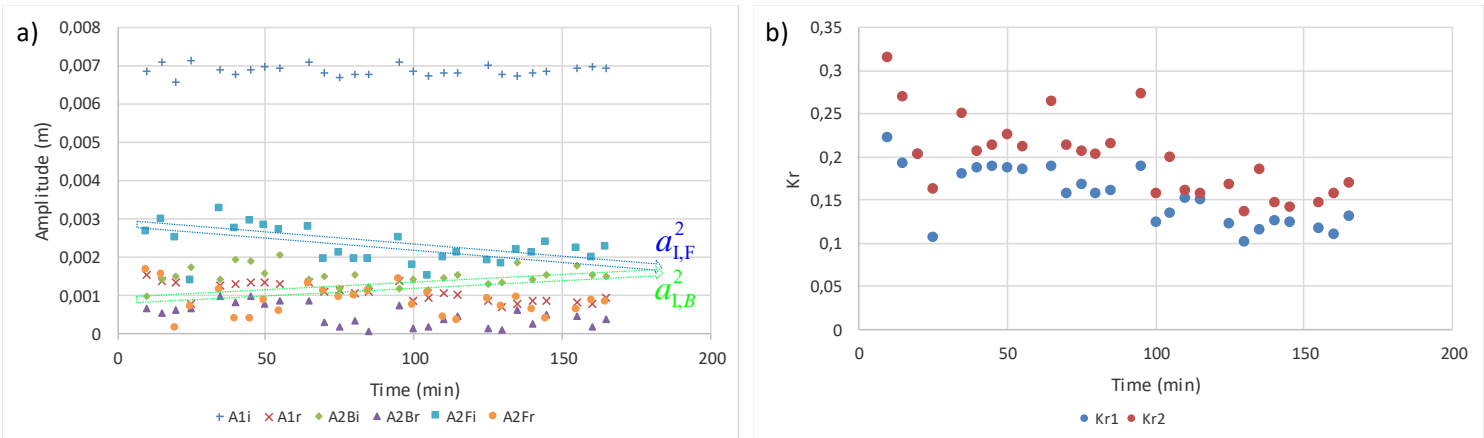

Figure 6. Evolution temporelle des coefficients de vagues (a) et de réflexion (b) du mur lors de l'essai 1.

La composante principale $a_{I}^{(1)}$, du signal est constante avec le temps; elle est entretenue par le mouvement régulier du batteur. Les composantes en réflexion marquent une diminution ; la composante $a_{I, B}^{(2)}$ enregistre une augmentation observable. Ces évolutions expliquent la diminution du coefficient de réflexion, d'ordre 1 ou 2 , avec le temps ou le phénomène d'affouillement.

\section{Conclusions}




\section{Thème 2 - Dynamique sédimentaire}

Un nouvel montage expérimental a été réalisé pour des tests d'affouillements de mur de protection côtière. Une série de 20 tests sont présentés pour différentes conditions de vagues et de plage. Les résultats d'affouillement sont comparés à des formules empiriques. Globalement, les résultats confirment les tendances que prédisent la formule de MCDOUGAL et al (1996). Toutefois cette relation ne prédit pas la possibilité d'un dépôt, ce qui a été observé dans les études de PEARCE et al. (2006) et dans certains de nos cas. Enfin les formules portent sur la profondeur maximale et la cinétique est mal documentée.

Cette étude sera poursuivie par des essais complémentaires, des analyses plus fines sur les vitesses verticales de jet et le transfert modale dans la décomposition dynamique des vagues. Le cas d'un mur avec des macro-rugosités est envisagé pour de futurs tests. Un jeu de données sera par la suite extrait pour alimenter un code numérique.

\section{Remerciements}

Les auteurs remercient le Ministère des Transports du Québec (MTQ) et le Conseil de Recherches en Sciences Naturelles et en Génie du Canada (NSERC-CRSNG) pour leurs financements (projet MTQ-IMAGE-R829.1) et (projet ECCOH-RGPIN-2018-06777).

\section{Références bibliographiques}

FOWLER J.E. (1992). Scour problems and methods for prediction of maximum scour at vertical seawalls, Coastal Engineering Research Center Vicksburg MS, 70 p.

HERBICH J.B., VAN WEELE B. (1965). Scour of flat sand beaches due to wave action in front of sea walls, Proceedings Santa Barbara specialty conference, ASCE, pp 705-726. LIN C.-Y., HUANG C.-J. (2004). Decomposition of incident and reflected higher harmonic waves using four wave gauges, Coastal engineering, Vol. 51(5-6), pp 395-406. https://doi.org/10.1016/j.coastaleng.2004.04.004

MCDOUGAL W.G., KRAUS N.C., AJIWIBOWO H. (1996). The effects of seawalls on the beach: Part II, Numerical modelling of SUPERTANK seawall tests, J. Coastal Research, Vol. 12(3), pp 702-713.

PEARCE A.M.C., SUTHERLAND J.S., OBHRAI C., MULLER G., RYCROFT D., WHITEHOUSE R.J.S. (2006). Scour at a seawall - Field measurements and laboratory modelling, Proceedings of ICCE, pp 2378-2390. https://doi.org/10.1142/9789812709554 0201

PEDOCCHI F., MARTIN J.E., GARCÍA M.H. (2008). Inexpensive fluorescent particles for large-scale experiments using particle image velocimetry, Experiments in Fluids, Vol. 45(1), pp 183-186. https://doi.org/10.1007/s00348-008-0516-2

SONG W.O., SCHILLER R.E. (1973). Experimental studies of beach scour due to wave action, COE Report. 166, TAMU-SG-73-211, Texas A\&M University.

WHITEHOUSE R. (1998). Scour at marine structures: A manual for practical applications, Thomas Telford, $198 \mathrm{p}$. 